\title{
Relationship between osteoarthritis findings in knee radiography and meniscus lesion in magnetic resonance imaging in symptomatic knee pain cases
}

\author{
Melike Elif Kalfaoglu, Zeliha Cosgun, Emine Dagistan \\ Department of Radiology, Bolu Abant Izzet Baysal University, Faculty of Medicine, Bolu, Turkey
}

\section{ABSTRACT}

\begin{abstract}
Aim: Knee osteoarthritis is a very common joint disease in the community. However, some meniscus lesions are asymptomatic. Studies show that a significant number of individuals with knee pain without radiographic osteoarthritis findings show meniscus injury on magnetic resonance imaging (MRI). Our study aimed to evaluate the relationship between meniscus lesions and the presence of radiographic knee osteoarthritis in individuals over 50 years of age with knee pain complaints.

Methods: Radiographic and MRI results of two hundred and forty patients who applied to our hospital with the complaint of knee pain between August 2018 and January 2020 were analyzed. Radiographic grading for knee osteoarthritis was performed using the Kellgren Lawrence scale. Classification of meniscus lesions in MRI was made as per the criteria defined by the British Knee Meniscus Surgery Association. Intergroup results were evaluated statistically.
\end{abstract}

Results: Osteoarthritis was detected in 110 (45.8\%) of 240 knee radiographs. In 78.3\% of all cases, meniscus lesions were detected in $96.4 \%$ of those with osteoarthritis and $63 \%$ of those without osteoarthritis. In patients with osteoarthritis, the prevalence of surgically targeted and possible target lesions was found to be significantly higher than those with no arthritis findings.

Conclusions: According to our study results, meniscus lesions were found quite common in individuals with knee pain, especially those with osteoarthritis. Particularly in patients with radiographic osteoarthritis findings, surgical targets and possible target meniscus lesions were more common than those without osteoarthritis findings. Therefore, MRI, in addition to direct radiography, should not be overlooked when determining treatment.

Key words: Osteoarthritis, meniscus lesions, radiography, magnetic resonance imaging.

Dr. Melike Elif Kalfaoglu

Department of Radiology, Bolu Abant Izzet Baysal

University, Faculty of Medicine, Bolu, Turkey

E-mail: melikekalfaoglu@hotmail.com

Received: 2021-05-18

Accepted: 2021-06-16/ Published online: 2021-07-01

\section{Introduction}

Osteoarthritis (OA) is very common in the population. It is a significant cause of morbidity, especially in the elderly population, and the knee is one of the most commonly affected joints. Osteoarthritis causes loss of working time by making it difficult to do many daily activities and is among the top ten causes of disability [1,2]. Approximately 25\% of people over the age of 50 suffer from knee pain due to degenerative knee disease [3]. In daily practice, non-traumatic knee pain of a middleaged or elderly patient is often associated with knee OA. However, some meniscus lesions have been reported asymptomatic in this age group [4]. 
Extensive scientific research performed in recent years has revealed the anatomical, biomechanical, and functional importance of the meniscus in the knee joint. As a vital part of the joint, it acts to prevent the disruption and degeneration of articular cartilage and the onset and development of OA [6]. Studies show that an Magnetic resonance imaging (MRI) reveals meniscus injury in a significant number of individuals with knee pain without radiographic OA findings [6,7]. Menisci are rarely normal in patients with knee OA, and there is a strong relationship between knee $\mathrm{OA}$ and meniscus pathologies. The relationship between meniscus damage and knee OA is complex. Meniscus lesions developing in a healthy knee may eventually lead to OA. Meniscus lesions may result from knee OA or might be the cause $[8,9]$. The distinctive structural feature of OA is the loss of joint cartilage. Although there is a weak correlation between the findings and clinical symptoms, direct radiographs are the first choice for OA imaging, including followup. However, since the narrowing of the joint distance is an indirect indicator of the state of the articular cartilage and radiographs are twodimensional, they provide limited information in evaluating three-dimensional joint structures $[10,11]$. Studies have shown that OA is a disorder involving other knee joint tissues, such as subchondral bone, ligaments, synovial membrane, muscle, and meniscus. Therefore, with its cross-sectional imaging, high spatial resolution, high tissue contrast, and multiplanar imaging features, MRI allows the evaluation of all pathologies belonging to the structures that make up the knee joint $[12,13]$.

Our study aimed to evaluate the relationship between OA findings in knee radiography and meniscus lesions in MRI in symptomatic knee pain cases.

\section{Materials and methods}

The Abant Izzet Baysal University, Clinical Research Local Ethics Committee (27.10.2020: 2020/253) provided ethical approval. The examinations of 318 patients between the ages of 50 and 79 who had knee radiography and knee MRI and were admitted to our hospital between August 2018 and January 2020 with pain complaints were examined.

The study included 240 cases and excluded seventy-eight cases with a history of knee trauma ( $n=26)$, Body mass index (BMI) of $>30$ $(n=19)$, anterior cruciate ligament tear $(n=16)$, and previous knee surgery $(n=17)$. The demographic data of the patients were evaluated retrospectively using the PACS system (picture archiving and communication system) of our hospital. A radiologist with ten years of experience interpreted antero-posterior knee radiographs and knee MRIs obtained in standing positions.

Radiographic grading for knee OA was performed using the Kellgren Lawrence scale (KL) [14]. The levels were Grade 0: Normal; Grade 1: Suspected joint narrowing, possible osteophytic sharpening; Grade 2: Possible joint narrowing, marked osteophytes; Grade 3: Definite joint distance narrowing, mild sclerosis, small pseudocystic areas, multiple osteophytes; and Grade 4: Severe narrowing of the joint distance, intense sclerosis and irregularity in the joint surface, and large osteophytes.

Cases with a degree of $\geq 2$ according to $\mathrm{KL}$ were considered positive for OA. All MRI examinations were performed with a $1.5 \mathrm{~T}$ MRI device (Symphony; Siemens, Erlangen, Germany). Section thickness was $4 \mathrm{~mm}$. Images were obtained as T1-weighted images (repeat time $(\mathrm{TR}) /$ echo time $(\mathrm{TE})=473 / 14 \mathrm{~ms}$ ), T2-weighted images $(\mathrm{TR} / \mathrm{TE}=3760 / 68 \mathrm{~ms})$, 
and PD-weighted images $\quad(\mathrm{TR} / \mathrm{TE}=2760 / 23$ $\mathrm{ms})$.

Classification of meniscus lesions in MRI was made as per the criteria defined by the British Knee Meniscus Surgery Association. Accordingly, meniscus lesions were classified as: 1) target lesion (surgically treatable): bucket handle tear, displaced meniscus tear, meniscus root insufficiency; 2) possible target lesion (unclear; may require surgical treatment): radial tear, horizontal tear \pm cyst, complex tear, short longitudinal tear; and

3) non-target lesion (possibly untreatable, meniscus surgery is not necessary): contour abnormality, isolated meniscus extrusion.

\section{Statistical analysis}

Data were analyzed using the SPSS 15.0 program. The Mann-Whitney U test compared the numerical data of the two independent groups, and the Pearson chi-square test evaluated the relationship between categorical variables. A p-value of $<0.05$ was considered statistically significant.

\section{Results}

A total of 240 patients were involved in the study. One hundred forty-nine of the patients were female, and 91 were male. The mean age of those with radiographic findings of OA was $60 \pm 6.7$ years, and the mean age of those without OA findings was $54 \pm 5.4$ years.
Osteoarthritis was detected in $110(45.8 \%)$ of 240 knee radiographs. We detected meniscus lesions in $96.4 \%(n=106)$ of those with OA and $63 \%(n=82)$ of those without OA, in $78.3 \%$ of all cases (Table 1). In patients with knee OA, the prevalence of surgically targeted and possible target lesions was significantly higher than those with no OA findings (Figure 1). Target and possible target lesions were detected in approximately $49 \%$ of the cases without radiographically discovered $\mathrm{OA}$. The most common lesions observed were horizontal and complex meniscus tears, respectively (Figure 2 and 3).

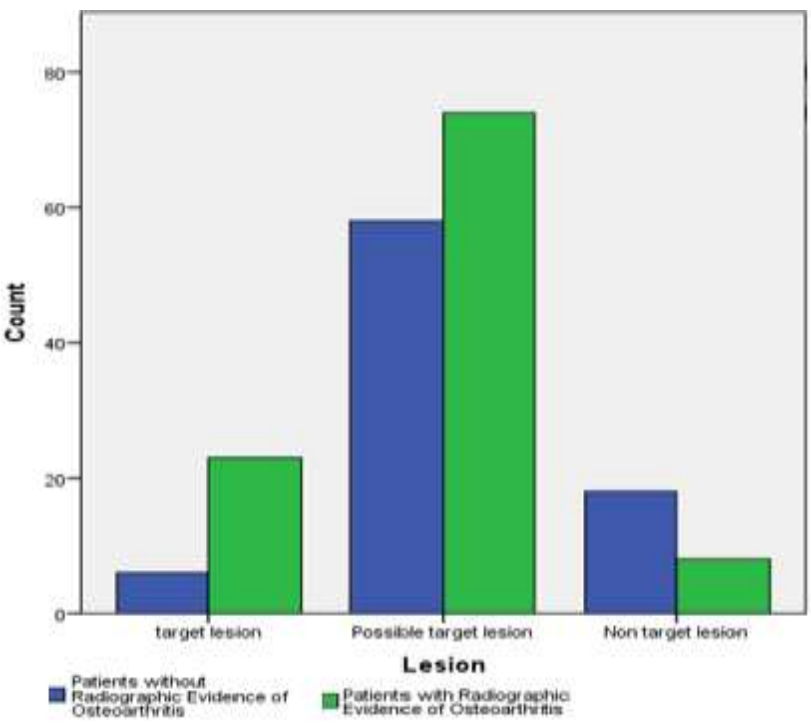

Figure 1. The prevalence of the meniscus lesion types according to the presence or absence of radiographic evidence of the knee osteoarthritis.

Table 1. The distribution of the meniscal lesions according to whether there is radiographic evidence of the knee osteoarthritis or not.

\begin{tabular}{|l|c|c|c|}
\hline \multicolumn{1}{|c|}{$\begin{array}{c}\text { MRI meniscus } \\
\text { lesion types }\end{array}$} & $\begin{array}{c}\text { Patients with Radiographic } \\
\text { evidence of osteoarthritis } \\
(\mathbf{n = 1 1 0})\end{array}$ & $\begin{array}{c}\text { Patients without Radiographic } \\
\text { evidence of osteoarthritis } \\
(\mathbf{n = 1 3 0}\end{array}$ & $\boldsymbol{P}$ Value \\
\hline Target lesion & $23(20.9)$ & $6(4.6)$ & 0.001 \\
\hline Possible target lesion & $74(67.3)$ & $58(44.6)$ & 0.001 \\
\hline Non-target lesion & $9(8.2)$ & $18(13.8)$ & 0.166 \\
\hline Total & $106(96.4)$ & $82(63.07)$ & 0.001 \\
\hline
\end{tabular}




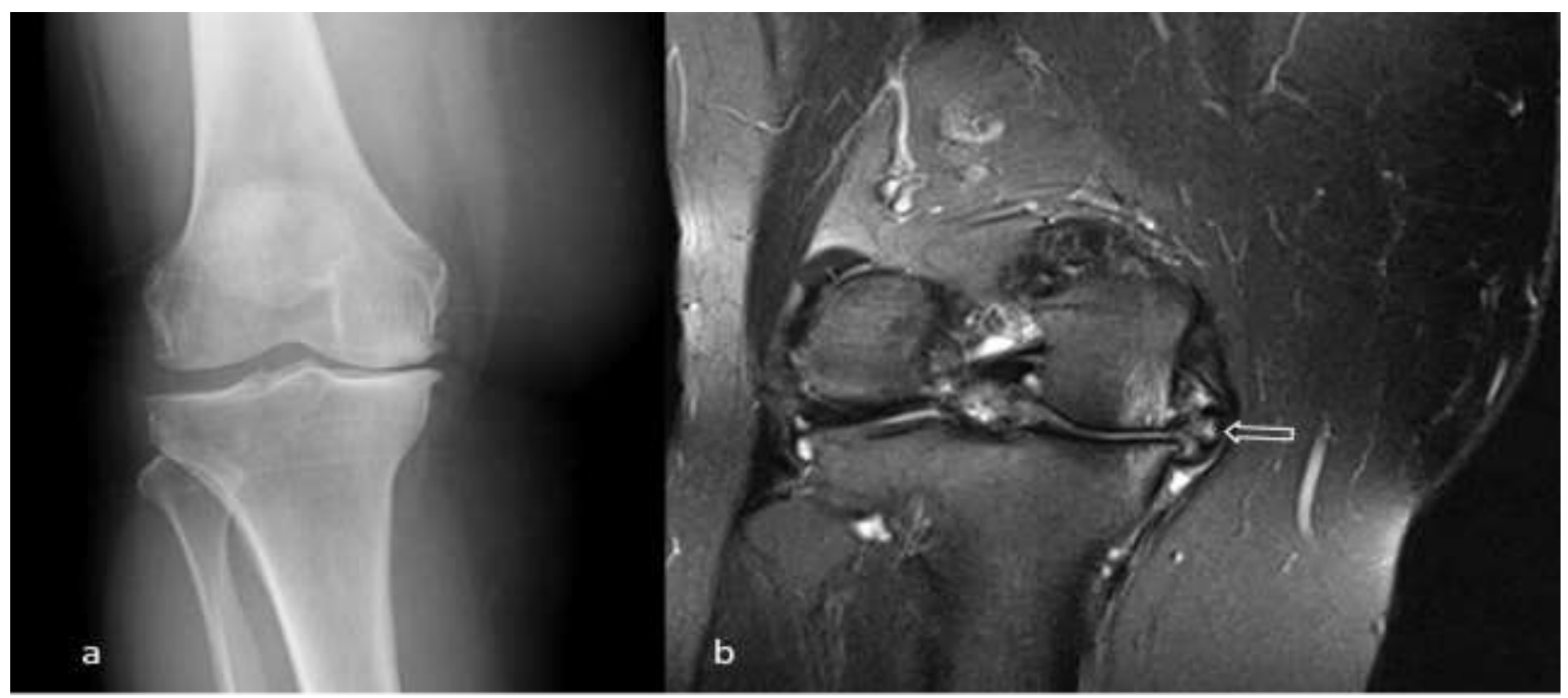

Figure 2. Anteroposterior radiograph of the right knee of a 54-year-old woman with knee pain shows Kellgren Lawrence grade 3 changes (a). A displaced medial meniscus tear (open arrow) is seen on the coronal T2 weighted magnetic resonance image of the same patient (b).

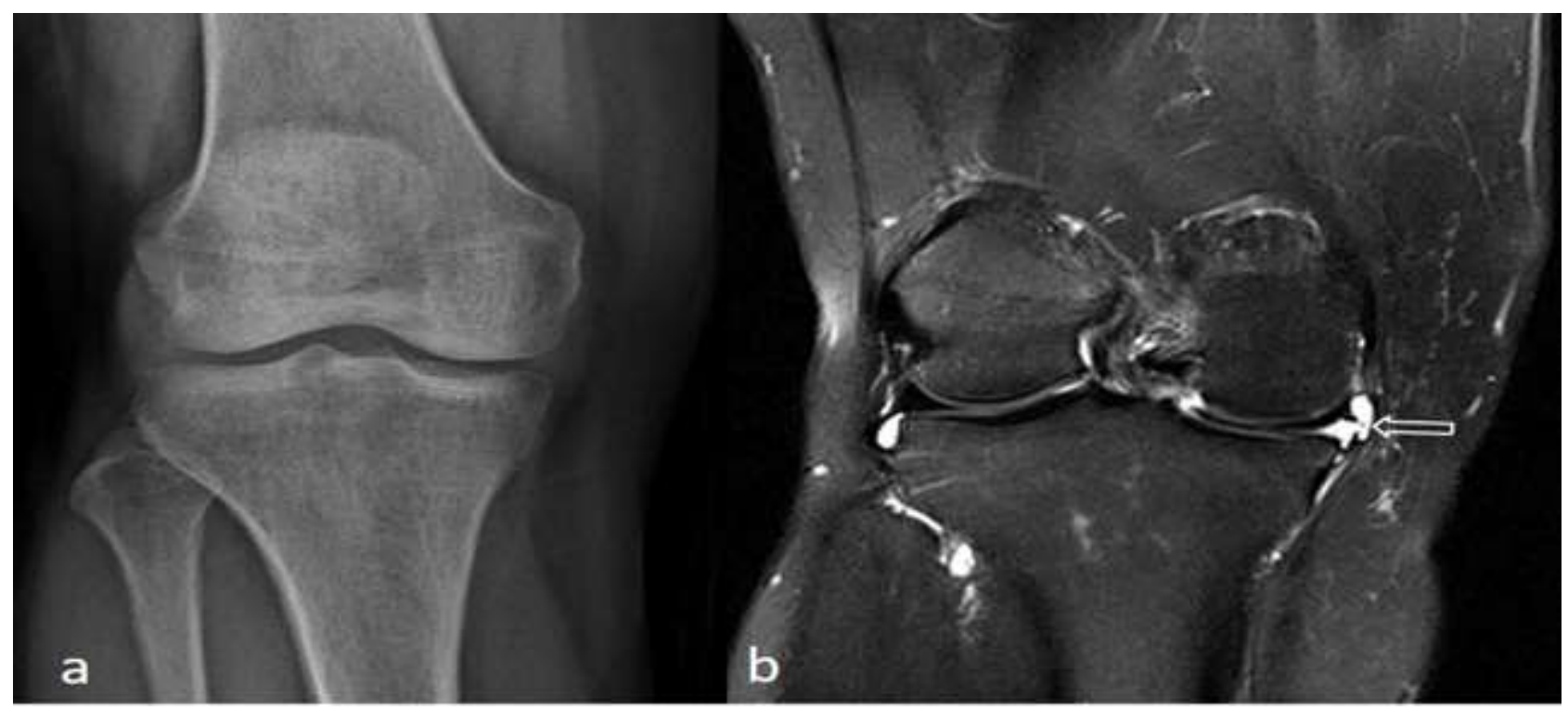

Figure 3. Anteroposterior radiograph of the right knee of a 52-year-old woman with knee pain shows Kellgren Lawrence grade 1 changes (a). A Horizontal tear and meniscus cyst (open arrow) are seen on the coronal T2 weighted magnetic resonance image of the same patient $(\mathbf{b})$.

\section{Discussion}

Our study found the prevalence of surgical target and possible target lesions to be higher in patients over 50 years of age with radiographic knee OA compared to those without OA. The most common target lesion observed in patients with and without $\mathrm{OA}$ was meniscus root insufficiency, and possible target lesions were horizontal and complex meniscus tears, respectively. The rate of meniscus tears was high in both groups. These findings show us that MRI should be performed in addition to direct radiography when determining treatment. Root tear was the most common lesion we found, with $13.6 \%$ of those having radiographic OA findings. Root tears have been shown to 
disrupt knee joint dynamics by causing meniscus extrusion and OA [16]. The low incidence of root tears in our group without radiographic OA supports this finding.

Meniscus tears are asymptomatic in elderly individuals and quite common in those with knee OA and correlated with the degree of OA. Studies have shown that meniscus tears do not significantly affect pain and function in patients with OA. Meniscus tears have been reported in up to $65 \%$ of asymptomatic individuals evaluated by MRI [17]. Controversial results have been obtained regarding whether knee pain is secondary to OA or meniscus tear. Englund et al. showed that meniscus lesions are quite common in individuals with knee pain, especially those with $\mathrm{OA}$, increasing with age $[4,17]$. Our study detected meniscus lesions in $63 \%$ of those without OA and $96 \%$ of those with OA. According to the literature, the reason for our higher meniscus lesion rates may be our higher mean age. These results suggest that knee OA increases meniscus injury.

The frequency of meniscus injuries in the general population is approximately $12-14 \%$. Although traumatic meniscus tears are associated with sports injuries in younger age groups, age-related degenerative tears constitute $30 \%$ of meniscus tears and, unlike acute tears, may be unrelated to trauma [18]. Meniscus tears can be treated with conservative treatment, surgical repair, or partial or complete meniscectomy [19]. The general tendency is to apply physical therapy/rehabilitation and nonsurgical treatment, especially in the OA group. It is essential to define the morphology of the tear well with MRI as it will affect the decision regarding surgical treatment.

While surgical repair is possible in horizontal tears, meniscus root tears - which are relatively rare in the general population - are lesions that constitute approximately $10.1 \%$ of arthroscopic meniscectomies. An MRI is usually required to identify root tears. In our study, the most common target lesion we detected in patients with radiographic OA was meniscus root tear, and it was observed in $13.6 \%$ of the patients. Root tears may cause extrusion of the meniscus body relative to the joint and also significantly weaken the ability of the meniscus to dissipate circumferential stress. As a result, it increases contact forces along the joint, causing OA and predisposing the patient to the development of cartilage damage [16]. In our study, statistically more root tears were observed in patients with OA, which is consistent with the literature's information.

The literature reports that meniscus extrusion is associated with the development and progression of OA [20].

Studies show that meniscus extrusion is evident in middle-aged individuals without knee OA [21]. These results suggest that meniscus extrusion is common not only in individuals with knee OA but also in those without OA findings. In line with these data, we found that meniscus extrusion was the most common nontarget lesion in our patient population, both in the group with and without knee OA.

The most important limitation of this study is that the imaging findings were not evaluated together with clinical findings, and the imaging findings were evaluated by a single radiologist. Therefore, more comprehensive studies that evaluate clinical and radiological findings together are needed to support the findings of the present study.

\section{Conclusions}

The menisci play a critical role in the knee joint. Meniscus tears can cause knee OA, and knee OA can also cause a meniscus tear. Although radiographs are the first imaging methods used to evaluate knee OA, MRI is required for 
evaluating meniscus lesions and defining tear morphology. A meniscus lesion detected in a middle-aged or elderly patient may be an indication of early-stage OA. There are different approaches in the treatment of meniscus tears depending on the type of tear. Detection of meniscus lesions on MRI and a good definition of the tear type constitute critical parameters in determining conservative or surgical treatment.

Magnetic resonance imaging of the knee is widely used to diagnose meniscus injury, and clinicians often use MRI findings as a factor in determining whether a person should undergo an arthroscopic meniscectomy. However, there is a lack of epidemiological data on the prevalence of meniscus tears in the general population.

Funding: The author(s) received no financial support for the research, authorship, and/or publication of this article.

Conflict of Interest: The authors declare that they have no conflict of interest.

\section{Ethical statement:}

The study was approved by Local Clinical Research Ethics Committee (Decision number: 2020/253, Date: 27.10.2020), and written informed consent was obtained from each subject.

\section{Open Access Statement}

This is an open access journal which means that all content is freely available without charge to the user or his/her institution under the terms of the Creative Commons Attribution NonCommercial License

(http://creativecommons.org/licenses/bync/4.0). Users are allowed to read, download, copy, distribute, print, search, or link to the full texts of the articles, without asking prior permission from the publisher or the author.

\section{References}

[1]Murray CJL, Vos $\mathrm{T}$, Lozano $\mathrm{R}$, et al. Disability-adjusted life years (DALYs) for 291 diseases and injuries in 21 regions, 1990-2010: A systematic analysis for the Global Burden of Disease Study 2010. Lancet. 2012;380(9859):2197-223.

[2]Bortoluzzi A, Furini F, Scirè CA. Osteoarthritis and its management Epidemiology, nutritional aspects, and environmental factors. Autoimmun Rev. 2018;17(11):1097-104.

[3]Nguyen USDT, Zhang Y, Zhu Y, et al. Increasing prevalence of knee pain and symptomatic knee osteoarthritis: Survey and cohort data. Ann Intern Med. 2011;155(11):725-32.

[4]Englund M, Guermazi A, Gale D, et al. Incidental Meniscus Findings on Knee MRI in Middle-Aged and Elderly Persons. N Engl J Med. 2008;359(11):1108-15.

[5]Makris EA, Hadidi P, Athanasiou KA. The knee meniscus: Structure-function, pathophysiology, current repair techniques, and prospects for regeneration. Biomaterials. 2011;32(30):7411-31.

[6]Guermazi A, Niu J, Hayashi D, et al. Prevalence of abnormalities in knees detected by MRI in adults without knee osteoarthritis: Population-based observational study (Framingham Osteoarthritis Study).

BMJ. 2012;345(7874).

[7]Kumm J, Turkiewicz A, Zhang F, et al. Structural abnormalities detected by knee magnetic resonance imaging are common in middle-aged subjects with and without risk factors for osteoarthritis. Acta Orthop. 2018;89(5):535-40.

[8]Chambers HG, Chambers RC. The Natural History of Meniscus Tears. J Pediatr Orthop. 2019;39(6):53-5. 
[9]Englund M, Guermazi A, Lohmander SL. The Role of the Meniscus in Knee Osteoarthritis: a Cause or Consequence? Radiol Clin North Am. 2009;47(4):703-12.

[10] Kornaat PR, Bloem JL, Ceulemans RY, et al. Osteoarthritis of the Knee: Association between Clinical Features and MR Imaging Findings. Radiology. 2006;239(3):811-7.

[11]Jacobson JA, Girish G, Jiang $Y$ et al. Radiographic Evaluation of Arthritis: Degenerative Joint Disease and Variations. Radiology. 2008;248(3):737-47.

[12] Hayes CW, Jamadar DA, Welch GW, et al. Osteoarthritis of the Knee: Comparison of MR Imaging Findings with Radiographic Severity Measurements and Pain in Middleaged Women. Radiology. 2005;237(3):9981007.

[13]Nguyen JG, Smet AA De, Graf BK, et al. MR Imaging-based Diagnosis and Classification of Meniscus Tears. RadioGraphics. 2014;34(1):981-99.

[14] Kellgren JH, Lawrence JS. Radiological Assessment of Osteo-Arthrosis. Ann Rheum Dis. 1957;(3):494-502.

[15] Abram SGF, Beard DJ, Price AJ, et al. The Knee National consensus on the definition, investigation, and classifi cation of meniscus lesions of the knee. The Knee. 2018;25(5):834-40.

[16] Russ P, Kilcoyne RF, Russ PD, et al. MR imaging patterns of displaced meniscus injuries of the knee. Am J Roentgenol. 1998;17(1):63-7.

[17] Bhattacharyya T, Gale D, Dewire P, et al. The clinical importance of meniscus tears demonstrated by magnetic resonance imaging in osteoarthritis of the knee. J Bone Jt Surg. 2003;85(1):4-9

[18]Fox AJS, Wanivenhaus F, Burge AJ, et al. The human meniscus : a review of anatomy, function, injury, and advances in treatment.
Clin Anat. 2014;28(2):269-87.

[19]Fox MG. MR imaging of the meniscus : review, current trends, and clinical implications. Radiol Clin North Am. 2007;45(6):1033-53.

[20]Guermazi A, Hayashi D, Jarraya M, et al. Medial posterior meniscus root tears are associated with development or worsening of medial tibiofemoral cartilage damage : the multicenter osteoarthritis study. Radiology. 2013;268(3):814-21.

[21] Svensson F, Felson DT, Zhang F, et al. Meniscus body extrusion and cartilage coverage in middle-aged and elderly without radiographic knee osteoarthritis. Eur Radiol. 2019;29(4):1848-54. 